\title{
H2AZ1 Gene
}

National Cancer Institute

\section{Source}

National Cancer Institute. H2AZ1 Gene. NCI Thesaurus. Code C126764.

This gene is involved in nucleosome formation. 